\title{
Facultative monophagy as a consequence of prior feeding experience: behavioral and physiological specialization in Colias philodice larvae
}

\author{
D.N. Karowe* \\ Department of Biology, University of Michigan, Ann Arbor, MI 48109, USA
}

\begin{abstract}
Summary. Although newly-emerged Colias philodice readily accept Medicago sativa, Melilotus alba, and Coronilla varia, fifth instar larvae reared on any single plant species display a highly significant induced feeding preference for their rearing host. Forced host-switching reveals that fifth instar relative growth rate (RGR) on $M$. sativa and $M$. alba is significantly reduced by prior feeding on either alternative host. Moreover, regardless of rearing diet, switching to a novel host during the fifth instar results in reduced $R G R$, relative consumption rate ( $R C R$ ), efficiency of conversion of digested food (ECD), and pupal weight. These results support the hypothesis that induction of feeding preference is an adaptive response that predisposes larvae to feed on the plant species they are most capable of utilizing for growth.
\end{abstract}

Key words: Herbivory - Colias - Preference induction Host-switching - Nutritional ecology

For herbivorous insects, the term "generalist" is commonly used to describe a species that, over its entire geographic range, feeds on a relatively diverse array of host plants. However, as argued by Fox and Morrow (1981), such species-level descriptions are potentially misleading because they may obscure variation that exists either among populations or among individuals within a population.

A number of recent studies of herbivorous insects have documented substantial within-population variation in the ability to use host tissue for growth. Although several studies have demonstrated a genetic basis for within-population variation in the ability to use a host (e.g. Gould 1979, 1983; Mitter et al. 1979; Jaenike and Grimaldi 1983; Hare and Kennedy 1986; Via 1986), the majority of within-population variation identified to date appears to be phenotypic (Jermy et al. 1968; Hanson 1976; Grabstein and Scriber 1982; Stanton 1983; Papaj 1986). In particular, an individual's prior feeding experience may strongly influence its subsequent ability to utilize different host plants.

In separate studies, prior feeding experience has been shown to influence either behavioral traits, including feeding preference (Jermy et al. 1968; Yamamoto 1974; Hanson 1976; Cassidy 1978; Barbosa et al. 1979; Dethier 1980),

\footnotetext{
* Present address and address for offprint requests: P.O.B. 8031 Wageningen Agricultural University, 6700 EH Wageningen, The Netherlands
}

or physiological traits, including the activity levels of digestive enzymes (Ishaaya and Swirski 1976) and mixed function oxidases (Brattsten et al. 1980; Ahmad 1983; Yu and Ing 1984). Two observations have led to the common inference that induction of feeding preference is an adaptation to avoid adverse effects associated with host-switching: (1) prior feeding on one host species frequently results in the induction of a feeding preference for that host (Hanson 1976), and (2) forced switching between host species often results in reduced growth (Schoonhoven and Meerman 1978; Scriber 1979; Grabstein and Scriber 1982). However, it is not yet possible to evaluate the adaptive value of preference induction because no single study has addressed the effect of prior feeding experience on both behavior and physiology of the same insect herbivore.

This study was designed to assess the effect of prior feeding experience on both host preference and physiological efficiency of host use in an herbivorous caterpillar. Colias philodice Latreille (Lepidoptera: Pieridae) feeds on a variety of herbaceous members of the Fabaceae. In southern Michigan, Medicago sativa L. (alfalfa) is a primary host for C. philodice, while Melilotus alba $\mathrm{L}$. (white sweet clover) is a secondary host, and Coronilla varia $\mathrm{L}$. (crown vetch) is a non-host (Karowe 1988). Three questions are addressed in this study: (1) Does prior feeding on any of these plant species result in an induced feeding preference in C. philodice?, (2) Is physiological efficiency of host use influenced by prior feeding on any of these species?, and (3) If so, do induced feeding preferences predispose larvae to select the plant species which they are physiologically most capable of utilizing for growth?

\section{Materials and methods}

\section{Experimental animals}

Colias philodice, the common or clouded sulfur butterfly, is distributed throughout most of the United States and contiguous southern Canada. At the species level, $C$. philodice is reported to feed on a wide variety of weedy legumes, including Medicago sativa (alfalfa), Trifolium repens $\mathrm{L}$. (white clover), T. pratense L. (red clover), Vicia spp. (vetch), and Lupinus spp. (lupines) (Klots 1951; Scott 1986). In Michigan, C. philodice also uses Melilotus alba (white sweet clover) as a secondary host (Karowe 1988).

All larvae used in these experiments were obtained from 17 females caught in June 1985 in southwestern Montmor- 
ency County, Michigan. Females were caught over a field of $M$. sativa and M. alba and were observed ovipositing on both species. Within $5 \mathrm{~h}$ of capture females were transported to the University of Michigan Biological Station, where they were allowed to oviposit on $M$. sativa in laboratory cages. Ten eggs from each female were placed on $M$. sativa, $M$. alba, and C. varia. Within each diet, the offspring of all females were reared together. Fresh food was provided every $48 \mathrm{~h}$.

\section{Acceptability of plant species to newly-emerged larvae}

To determine the acceptability of $M$. sativa, M. alba, and C. varia to newly-emerged larvae, immediately upon hatching 5 larvae were placed on approximately $5-\mathrm{cm}^{2}$ of leaf material (usually 2 leaves) of one plant species in a $5.5-\mathrm{cm}$ plastic Petri dish. These test larvae had not previously fed upon any plant material. Larvae that initiated feeding within $2 \mathrm{~h}$ were judged to have accepted that plant species as food. Between 41 and 88 larvae were tested on each species.

\section{The effect of feeding experience on host choice}

To determine whether previous feeding experience affects subsequent host choice, 20 freshly-molted fifth (ultimate) instar larvae from each rearing diet were chosen at random and placed individually into $10-\mathrm{cm}$ plastic Petri dishes lined with moist filter paper. Each Petri dish contained equal amounts ( $400 \mathrm{mg}$ fresh weight) of leaves of $M$. sativa, $M$. alba, and C. varia. Leaves of the three species were thoroughly mixed and larvae were allowed to feed freely for 26 to $32 \mathrm{~h}$. Plants were collected along paved country roads in western Washtenaw County, Michigan, and only uninjured young foliage was used. At the completion of each feeding trial, all remaining leaves were separated by species and dried to constant weight at $70^{\circ} \mathrm{C}$.

To estimate the dry weight of leaves used in preference tests, five 1-g samples of each plant species were weighed fresh, dried to constant weight at $70^{\circ} \mathrm{C}$, and reweighed. The amount of each species consumed was determined by subtracting the dry weight of uneaten leaves from the estimated initial dry weight of leaves provided. Percent dry matter did not differ among plant species; therefore, statistical analysis was performed on absolute, rather than proportional, amounts eaten. Analysis was restricted to trials in which the larva consumed no more than $70 \%$ of any species since, after this point, the larva was considered to have encountered a 2-way, rather than the intended 3-way, choice test. However, this resulted in the exclusion of only 4 of 60 trials.

All rearing and testing of larvae was conducted in an environmental chamber with a $16: 8 \mathrm{~L}: \mathrm{D}$ cycle and a corresponding $29^{\circ}: 19^{\circ} \mathrm{C}$ temperature cycle.

\section{The effect of feeding experience on physiology of host use}

To determine whether previous feeding experience affects the physiology of host use, 48 freshly-molted fifth instar larvae from each rearing diet were divided into 3 equal groups and given either M. sativa, M. alba, or C. varia as the test diet. Since C.philodice shows diurnal periodicity in consumption rate (personal observation), only larvae molting to the fifth instar between 8 a.m. and 11 a.m. were used. Thus, all test larvae started with 13 to $16 \mathrm{~h}$ of photo-
Table 1. Acceptability of $M$. sativa, $M$. alba, and C. varia to newlyemerged C.philodice larvae. Acceptability does not differ among plant species $\left(X^{2}=1.67, P>0.4\right)$

\begin{tabular}{llll}
\hline Plant Species & $\begin{array}{l}\text { No. Larvae } \\
\text { Accepting }\end{array}$ & $\begin{array}{l}\text { No. Larvae } \\
\text { Rejecting }\end{array}$ & $\begin{array}{l}\text { Percent } \\
\text { Acceptance }\end{array}$ \\
\hline M. sativa & 82 & 6 & $93.2 \mathrm{a}$ \\
M. alba & 70 & 10 & $87.5 \mathrm{a}$ \\
C. varia & 71 & 9 & $88.8 \mathrm{a}$ \\
\hline
\end{tabular}

Table 2. Feeding preferences of fifth instar $C$. philodice larvae as a function of plant species consumed in earlier instars. Values are mean amount (grams dry weight) eaten in three-way choice tests, with standard deviations in parentheses. Means followed by different letters are significantly different at $P<0.05$ by LSD

\begin{tabular}{lccc}
\hline Species Eaten & \multicolumn{3}{l}{ Rearing Diet } \\
\cline { 2 - 4 } & $\begin{array}{l}\text { M. sativa } \\
(n=19)\end{array}$ & $\begin{array}{c}\text { M. alba } \\
(n=18)\end{array}$ & $\begin{array}{c}\text { C. varia } \\
(n=19)\end{array}$ \\
\hline M. sativa & $0.0297 \mathrm{a}$ & $0.0149 \mathrm{a}$ & $0.0149 \mathrm{a}$ \\
M. alba & $(0.0108)$ & $(0.0111)$ & $(0.0095)$ \\
& $0.0098 \mathrm{~b}$ & $0.0301 \mathrm{~b}$ & $0.0084 \mathrm{~b}$ \\
C. varia & $(0.0072)$ & $(0.0125)$ & $(0.0098)$ \\
& $0.0133 \mathrm{~b}$ & $0.0127 \mathrm{a}$ & $0.0233 \mathrm{c}$ \\
& $(0.0087)$ & $(0.0114)$ & $(0.0098)$ \\
\hline
\end{tabular}

phase remaining in their first $24 \mathrm{~h}$ cycle. Larvae molting after 11 a.m. ( 25 to 29 per diet) were sacrificed to yield a wet weight/dry weight conversion factor for test larvae from each rearing diet. To avoid placing a disproportionate number of fast- or slow-growing larvae on any test diet, the first, fourth, seventh, tenth, etc. larvae from any rearing diet to molt to the fifth instar were placed on $M$. sativa; the second, fifth, eighth, etc. were placed on M. alba; and the third, sixth, ninth, etc. were placed on C. varia.

Standard quantitative nutritional indices (Waldbauer 1968) were calculated for each of the 17 larvae from each rearing diet on each test diet. Each larva was weighed at the onset of the fifth instar and placed in a $5.5-\mathrm{cm}$ plastic Petri dish lined with moist filter paper that contained a weighed amount of leaves of $M$. sativa, $M$. alba, or C. varia. Fresh food was provided every $48 \mathrm{~h}$ or more often if necessary. To estimate the dry weight of the food provided, five 1-gm samples were weighed wet, dried to constant weight at $70^{\circ} \mathrm{C}$, and reweighed. Since nutritional indices are sensitive to error inherent in estimating the dry weight of food provided (Schmidt and Reese 1986), larvae were given only slightly more food than they would eat in $48 \mathrm{~h}$.

Immediately upon pupating, each larva was weighed, then dried to constant weight at $70^{\circ} \mathrm{C}$. For each larva, relative growth rate (RGR), relative consumption rate (RCR), approximate digestibility (AD) and efficiency of conversion of digested food (ECD) were calculated as described by Waldbauer (1968):

$$
\begin{aligned}
& \mathrm{RGR}=\frac{\text { larval weight gained }}{\text { average larval weight during instar } \times \text { days }} \\
& \mathrm{RCR}=\frac{\text { weight of food ingested }}{\text { average larval weight during instar } \times \text { days }}
\end{aligned}
$$


Table 3. a Performance of fifth instar C. philodice larvae on $M$. sativa as a function of plant species consumed in earlier instars. All larvae were fed $M$. sativa during the ultimate instar but had been reared through the penultimate instar on $M$. sativa, $M$. alba, or C. varia. Mean values are given, with standard deviations in parentheses. Means followed by different letters are significantly different at $P<0.05$ by LSD

\begin{tabular}{|c|c|c|c|c|c|}
\hline Rearing Diet & $n$ & RGR & RCR & $\mathrm{ECD}$ & $\mathrm{AD}$ \\
\hline M. sativa & 17 & $\begin{array}{c}0.262 \mathrm{a} \\
(0.067)\end{array}$ & $\begin{array}{l}1.52 \mathrm{a} \\
(0.29)\end{array}$ & $\begin{array}{c}0.289 \mathrm{a} \\
(0.060)\end{array}$ & $\begin{array}{c}0.598 \mathrm{a} \\
(0.053)\end{array}$ \\
\hline M. alba & 16 & $\begin{array}{l}0.112 \mathrm{~b} \\
(0.085)\end{array}$ & $\begin{array}{l}1.23 b \\
(0.25)\end{array}$ & $\begin{array}{l}0.145 \mathrm{~b} \\
(0.106)\end{array}$ & $\begin{array}{c}0.654 \mathrm{~b} \\
(0.074)\end{array}$ \\
\hline \multirow[t]{2}{*}{ C. varia } & 15 & $\begin{array}{l}0.161 \mathrm{~b} \\
(0.113)\end{array}$ & $\begin{array}{l}1.57 \mathrm{a} \\
(0.39)\end{array}$ & $\begin{array}{l}0.173 \mathrm{~b} \\
(0.124)\end{array}$ & $\begin{array}{l}0.631 \mathrm{ab} \\
(0.110)\end{array}$ \\
\hline & \multicolumn{2}{|c|}{$\begin{array}{l}\text { Pupal } \\
\text { Weight } \\
\text { (gm dry) }\end{array}$} & \multicolumn{2}{|l|}{$\begin{array}{l}\text { Instar } \\
\text { Duration } \\
\text { (h) }\end{array}$} & $\begin{array}{l}\text { Total Food } \\
\text { Consumed } \\
\text { (gm dry) }\end{array}$ \\
\hline M. sativa & \multicolumn{2}{|c|}{$\begin{array}{c}0.029 \mathrm{a} \\
(0.012)\end{array}$} & $\begin{array}{l}103.8 \mathrm{a} \\
(37.7)\end{array}$ & \multicolumn{2}{|c|}{$\begin{array}{c}0.125 \mathrm{a} \\
(0.062)\end{array}$} \\
\hline M. alba & \multicolumn{2}{|c|}{$\begin{array}{c}0.016 \mathrm{~b} \\
(0.010)\end{array}$} & $\begin{array}{l}140.8 \mathrm{~b} \\
(41.1)\end{array}$ & \multicolumn{2}{|c|}{$\begin{array}{c}0.089 \mathrm{a} \\
(0.062)\end{array}$} \\
\hline C. varia & \multicolumn{2}{|c|}{$\begin{array}{l}0.017 \mathrm{~b} \\
(0.011)\end{array}$} & $\begin{array}{l}122.3 \mathrm{a} \mathrm{b} \\
(53.0)\end{array}$ & \multicolumn{2}{|r|}{$\begin{array}{c}0.090 \mathrm{a} \\
(0.060)\end{array}$} \\
\hline
\end{tabular}

b Performance of fifth instar $C$. philodice larvae on $M$, alba as a function of plant species consumed in earlier instars. All larvae were fed $M$. alba during the ultimate instar but had been reared through the penultimate instar on $M$. sativa, $M$. alba, or C. varia. Mean values are given, with standard deviations in parentheses. Means followed by different letters are significantly different at $P<0.05$ by LSD

\begin{tabular}{|c|c|c|c|c|c|}
\hline Rearing Diet & $n$ & RGR & $\mathrm{RCR}$ & $\mathrm{ECD}$ & $\mathrm{AD}$ \\
\hline M. sativa & 15 & $\begin{array}{c}0.082 \mathrm{a} \\
(0.099)\end{array}$ & $\begin{array}{l}1.22 \mathrm{a} \\
(0.29)\end{array}$ & $\begin{array}{c}0.101 \mathrm{a} \\
(0.120)\end{array}$ & $\begin{array}{c}0.701 \mathrm{a} \\
(0.041)\end{array}$ \\
\hline M. alba & 16 & $\begin{array}{l}0.306 \mathrm{~b} \\
(0.138)\end{array}$ & $\begin{array}{l}1.77 b \\
(0.40)\end{array}$ & $\begin{array}{c}0.279 b \\
(0.085)\end{array}$ & $\begin{array}{c}0.599 \mathrm{~b} \\
(0.027)\end{array}$ \\
\hline \multirow[t]{2}{*}{ C. varia } & 17 & $\begin{array}{c}0.115 \mathrm{c} \\
((0.082)\end{array}$ & $\begin{array}{l}1.17 \mathrm{a} \\
(0.26)\end{array}$ & $\begin{array}{c}0.148 \mathrm{c} \\
(0.085)\end{array}$ & $\begin{array}{c}0.646 \mathrm{c} \\
(0.046)\end{array}$ \\
\hline & \multicolumn{2}{|c|}{$\begin{array}{l}\text { Pupal } \\
\text { Weight } \\
\text { (gm dry) }\end{array}$} & $\begin{array}{l}\text { Instar } \\
\text { Duration } \\
\text { (h) }\end{array}$ & \multicolumn{2}{|r|}{$\begin{array}{l}\text { Total Food } \\
\text { Consumed } \\
\text { (gm dry) }\end{array}$} \\
\hline M. sativa & \multicolumn{2}{|c|}{$\begin{array}{c}0.014 \mathrm{a} \\
(0.013)\end{array}$} & $\begin{array}{l}149.4 \mathrm{a} \\
(60.5)\end{array}$ & \multicolumn{2}{|r|}{$\begin{array}{c}0.081 \mathrm{a} \\
(0.053)\end{array}$} \\
\hline M. alba & \multicolumn{2}{|c|}{$\begin{array}{l}0.034 \mathrm{~b} \\
(0.008)\end{array}$} & $\begin{array}{l}123.5 \mathrm{a} \\
(67.3)\end{array}$ & \multicolumn{2}{|c|}{$\begin{array}{l}0.162 \mathrm{~b} \\
(0.030)\end{array}$} \\
\hline C. varia & \multicolumn{2}{|c|}{$\begin{array}{c}0.016 \mathrm{a} \\
(0.009)\end{array}$} & $\begin{array}{l}168.4 \mathrm{a} \\
(69.3)\end{array}$ & \multicolumn{2}{|c|}{$\begin{array}{c}0.091 \mathrm{a} \\
(0.043)\end{array}$} \\
\hline
\end{tabular}

$\mathrm{AD}=\frac{\text { weight } \text { of food ingested }- \text { weight of frass }}{\text { weight of food ingested }}$

$$
\mathrm{ECD}=\frac{\text { larval weight gained }}{\text { weight of food ingested }- \text { weight of frass }}
$$

c Performance of fifth instar C. philodice larvae on C. varia as a function of plant species consumed in earlier instars. All larvae were fed $C$. varia during the ultimate instar but had been reared through the penultimate instar on $M$. sativa, $M$. alba, or $C$. varia. Mean values are given, with standard deviations in parentheses. Means followed by different letters are significantly different at $P<0.05$ by $\operatorname{LSD}$

\begin{tabular}{|c|c|c|c|c|c|}
\hline Rearing Diet & $n$ & RGR & $\mathrm{RCR}$ & $\mathrm{ECD}$ & $\mathrm{AD}$ \\
\hline M. sativa & 16 & $\begin{array}{c}0.190 \mathrm{a} \\
(0.072)\end{array}$ & $\begin{array}{c}1.23 \mathrm{a} \\
(0.19)\end{array}$ & $\begin{array}{c}0.231 \mathrm{a} \\
(0.082)\end{array}$ & $\begin{array}{c}0.671 \mathrm{a} \\
(0.034)\end{array}$ \\
\hline M. alba & 16 & $\begin{array}{l}0.200 \mathrm{a} \\
(0.084)\end{array}$ & $\begin{array}{l}1.28 \mathrm{a} \\
(0.34)\end{array}$ & $\begin{array}{c}0.242 \mathrm{a} \\
(0.093)\end{array}$ & $\begin{array}{c}0.667 \mathrm{a} \\
(0.058)\end{array}$ \\
\hline \multirow[t]{2}{*}{ C. varia } & 16 & $\begin{array}{c}0.216 \mathrm{a} \\
(0.112)\end{array}$ & $\begin{array}{c}1.31 \mathrm{a} \\
(0.28)\end{array}$ & $\begin{array}{c}0.248 \mathrm{a} \\
(0.110)\end{array}$ & $\begin{array}{c}0.645 \mathrm{a} \\
(0.051)\end{array}$ \\
\hline & \multicolumn{2}{|c|}{$\begin{array}{l}\text { Pupal } \\
\text { Weight } \\
\text { (gm dry) }\end{array}$} & \multicolumn{2}{|l|}{$\begin{array}{l}\text { Instar } \\
\text { Duration } \\
\text { (h) }\end{array}$} & $\begin{array}{l}\text { Total Food } \\
\text { Consumed } \\
\text { (gm dry) }\end{array}$ \\
\hline M. sativa & \multicolumn{2}{|c|}{$\begin{array}{c}0.025 \mathrm{a} \\
(0.008)\end{array}$} & $\begin{array}{l}148.6 \mathrm{a} \\
(23.6)\end{array}$ & & $\begin{array}{c}0.117 \mathrm{a} \\
(0.026)\end{array}$ \\
\hline M. alba & \multicolumn{2}{|c|}{$\begin{array}{l}0.025 \mathrm{a} \\
(0.010)\end{array}$} & $\begin{array}{l}126.4 \mathrm{a} \\
(46.2)\end{array}$ & & $\begin{array}{c}0.100 \mathrm{a} \\
(0.037)\end{array}$ \\
\hline C. varia & \multicolumn{2}{|c|}{$\begin{array}{l}0.026 \mathrm{a} \\
(0.013)\end{array}$} & $\begin{array}{l}147.8 \mathrm{a} \\
(77.1)\end{array}$ & & $\begin{array}{c}0.117 \mathrm{a} \\
(0.049)\end{array}$ \\
\hline
\end{tabular}

where average larval weight during the instar $=$ (initial weight + final weight) $/ 2$. All calculations were made on a dry weight basis.

\section{Results}

Acceptability of plant species to newly-emerged larvae

Newly-emerged larvae readily accepted all 3 plant species (Table 1); most larvae initiated feeding within 5 min. Acceptability did not differ among species $\left(X^{2}=1.67, P>0.4\right)$, ranging only from $93.2 \%$ for $M$. sativa to $87.5 \%$ for M. alba.

\section{The effect of prior feeding on subsequent host choice}

Larvae on all three plant species exhibited a strong induced feeding preference for the diet on which they were originally reared (Table 2). Larvae reared on $M$. sativa preferred this species more than $2: 1$ over either $M$. alba or C. varia. Similarly, larvae reared on $M$. alba preferred this species more than 2:1 over either $M$. sativa or C. varia. Larvae reared on C. varia preferred C. varia $1.5: 1$ over $M$. sativa and nearly $3: 1$ over $M$. alba.

Larvae reared on $M$. sativa did not consume significantly different amounts of $M$. alba and C. varia, nor did larvae reared on $M$. alba consume significantly different amounts of $M$. sativa and C.varia. However, larvae reared on C. varia consumed significantly more $M$. sativa than M. alba (Table 2).

\section{The effect of prior feeding experience on physiology of host use}

Comparisons among larvae with different rearing diets, but the same test diet clearly indicate that prior feeding experi- 
Table 4. a Fifth instar performance of $M$. sativa-reared larvae on $M$. sativa, M. alba, and C. varia. Means are given, with standard deviations in parentheses. Means followed by different letters are significantly different at $P<0.05$ by LSD

\begin{tabular}{|c|c|c|c|c|c|}
\hline Test Diet & $n$ & RGR & RCR & $\mathrm{ECD}$ & $\mathrm{AD}$ \\
\hline M. sativa & 17 & $\begin{array}{c}0.262 \mathrm{a} \\
(0.067)\end{array}$ & $\begin{array}{l}1.52 \mathrm{a} \\
(0.29)\end{array}$ & $\begin{array}{c}0.289 \mathrm{a} \\
(0.060)\end{array}$ & $\begin{array}{c}0.598 \mathrm{a} \\
(0.053)\end{array}$ \\
\hline M. alba & 15 & $\begin{array}{l}0.082 \mathrm{~b} \\
(0.099)\end{array}$ & $\begin{array}{l}1.22 \mathrm{~b} \\
(0.29)\end{array}$ & $\begin{array}{c}0.101 \mathrm{~b} \\
(0.120)\end{array}$ & $\begin{array}{c}0.701 b \\
(0.041)\end{array}$ \\
\hline \multirow[t]{2}{*}{ C. varia } & 16 & $\begin{array}{c}0.190 \mathrm{c} \\
(0.072)\end{array}$ & $\begin{array}{l}1.23 \mathrm{~b} \\
(0.19)\end{array}$ & $\begin{array}{l}0.231 \mathrm{~b} \\
(0.082)\end{array}$ & $\begin{array}{l}0.671 \mathrm{~b} \\
(0.034)\end{array}$ \\
\hline & \multicolumn{2}{|c|}{$\begin{array}{l}\text { Pupal } \\
\text { Weight } \\
\text { (gm dry) }\end{array}$} & \multicolumn{2}{|l|}{$\begin{array}{l}\text { Instar } \\
\text { Duration } \\
\text { (b) }\end{array}$} & $\begin{array}{l}\text { Total Food } \\
\text { Consumed } \\
\text { (gm dry) }\end{array}$ \\
\hline$M$. sativa & \multicolumn{2}{|c|}{$\begin{array}{c}0.029 \mathrm{a} \\
(0.012)\end{array}$} & $\begin{array}{l}103.8 \mathrm{a} \\
(37.7)\end{array}$ & \multicolumn{2}{|c|}{$\begin{array}{c}0.125 \mathrm{a} \\
(0.062)\end{array}$} \\
\hline M. alba & \multicolumn{2}{|c|}{$\begin{array}{c}0.014 \mathrm{~b} \\
(0.013)\end{array}$} & $\begin{array}{l}149.4 \mathrm{~b} \\
(60.5)\end{array}$ & \multicolumn{2}{|c|}{$\begin{array}{l}0.081 \mathrm{a} \\
(0.053)\end{array}$} \\
\hline C. varia & \multicolumn{2}{|c|}{$\begin{array}{l}0.025 \mathrm{a} \\
(0.008)\end{array}$} & $\begin{array}{l}148.6 \mathrm{~b} \\
(23.6)\end{array}$ & \multicolumn{2}{|c|}{$\begin{array}{c}0.117 \mathrm{a} \\
(0.026)\end{array}$} \\
\hline
\end{tabular}

b Fifth instar performance of $M$. alba-reared larvae on $M$. sativa, M. alba, and C. varia. Means are given, with standard deviations in parentheses. Means followed by different letters are significantly at $P<0.05$ by LSD

\begin{tabular}{|c|c|c|c|c|c|}
\hline Test Diet & $n$ & RGR & $\mathrm{RCR}$ & $\mathrm{ECD}$ & $\mathrm{AD}$ \\
\hline M. sativa & 16 & $\begin{array}{c}0.112 \mathrm{a} \\
(0.085)\end{array}$ & $\begin{array}{c}1.23 \mathrm{a} \\
(0.25)\end{array}$ & $\begin{array}{c}0.145 \mathrm{a} \\
(0.106)\end{array}$ & $\begin{array}{c}0.654 \mathrm{a} \\
(0.074)\end{array}$ \\
\hline M. alba & 16 & $\begin{array}{c}0.306 \mathrm{~b} \\
(0.138)\end{array}$ & $\begin{array}{l}1.77 \mathrm{~b} \\
(0.41)\end{array}$ & $\begin{array}{c}0.279 \mathrm{~b} \\
(0.085)\end{array}$ & $\begin{array}{c}0.599 \mathrm{~b} \\
(0.027)\end{array}$ \\
\hline \multirow[t]{2}{*}{ C. varia } & 16 & $\begin{array}{c}0.200 \mathrm{c} \\
(0.084)\end{array}$ & $\begin{array}{l}1.28 \mathrm{a} \\
(0.34)\end{array}$ & $\begin{array}{c}0.242 \mathrm{~b} \\
(0.093)\end{array}$ & $\begin{array}{c}0.667 \mathrm{a} \\
(0.058)\end{array}$ \\
\hline & \multicolumn{2}{|c|}{$\begin{array}{l}\text { Pupal } \\
\text { Weight } \\
\text { (gm dry) }\end{array}$} & $\begin{array}{l}\text { Instar } \\
\text { Duration } \\
\text { (h) }\end{array}$ & \multicolumn{2}{|r|}{$\begin{array}{l}\text { Total Food } \\
\text { Consumed } \\
\text { (gm dry) }\end{array}$} \\
\hline M. sativa & \multicolumn{2}{|c|}{$\begin{array}{c}0.016 \mathrm{a} \\
(0.010)\end{array}$} & $\begin{array}{l}140.8 \mathrm{a} \\
(41.1)\end{array}$ & \multicolumn{2}{|c|}{$\begin{array}{c}0.089 \mathrm{a} \\
(0.062)\end{array}$} \\
\hline M. alba & \multicolumn{2}{|c|}{$\begin{array}{l}0.034 \mathrm{~b} \\
(0.008)\end{array}$} & $\begin{array}{l}123.5 \mathrm{a} \\
(67.3)\end{array}$ & \multicolumn{2}{|c|}{$\begin{array}{l}0.162 \mathrm{~b} \\
(0.030)\end{array}$} \\
\hline C. varia & \multicolumn{2}{|c|}{$\begin{array}{l}0.025 \mathrm{c} \\
(0.010)\end{array}$} & $\begin{array}{l}126.4 \mathrm{a} \\
(46.2)\end{array}$ & \multicolumn{2}{|c|}{$\begin{array}{c}0.100 \mathrm{a} \\
(0.037)\end{array}$} \\
\hline
\end{tabular}

ence strongly influences the physiological ability of $C$. philodice larvae to utilize a particular host species. In nearly all cases, forces host-switching resulted in reduced larval growth that was attributable primarily to a reduced capacity to convert digested food into larval tissue (Table $3 a-c$ ).

Larvae forced to switch onto $M$. sativa during the ultimate instar, after feeding on either $M$. alba or $C$. varia through the penultimate instar, performed significantly worse than larvae fed continuously on $M$. sativa (Table $3 a$ ). Relative to larvae reared on $M$. sativa, larvae switched onto $M$. sativa from $M$. alba showed a $57 \%$ reduction in $\mathrm{RGR}$, a $50 \%$ reduction in $\mathrm{ECD}$, a $19 \%$ reduction in $\mathrm{RCR}$, a c Fifth instar performance of C. varia-reared larvae on $M$. sativa, $M$. alba, and $C$. varia. Means are given, with standard deviations in parentheses. Means followed by different letters are significantly different at $P<0.05$ by LSD

\begin{tabular}{llllll}
\hline Test Diet & $n$ & RGR & RCR & ECD & AD \\
\hline M. sativa & 15 & $0.161 \mathrm{ab}$ & $1.57 \mathrm{a}$ & $0.173 \mathrm{ab}$ & $0.631 \mathrm{a}$ \\
& & $(0.113)$ & $(0.39)$ & $(0.124)$ & $(0.110)$ \\
M. alba & 17 & $0.115 \mathrm{a}$ & $1.17 \mathrm{~b}$ & $0.148 \mathrm{a}$ & $0.646 \mathrm{a}$ \\
& & $(0.082)$ & $(0.26)$ & $(0.085)$ & $(0.046)$ \\
C. varia & 16 & $0.216 \mathrm{~b}$ & $1.31 \mathrm{ab}$ & $0.248 \mathrm{~b}$ & $0.645 \mathrm{a}$ \\
& & $(0.112)$ & $(0.28)$ & $(0.110)$ & $(0.050)$ \\
\hline & Pupal & Instar & Total Food \\
& Weight & Duration & Consumed \\
& $($ gm dry) & $(\mathrm{h})$ & $(\mathrm{gm} \mathrm{dry)}$ \\
\hline M. sativa & $0.017 \mathrm{a}$ & $122.3 \mathrm{a}$ & $0.090 \mathrm{a}$ \\
& $(0.011)$ & $(53.0)$ & $(0.060)$ \\
M. alba & $0.016 \mathrm{a}$ & $168.4 \mathrm{a}$ & $0.091 \mathrm{a}$ \\
& $(0.009)$ & $(69.3)$ & $(0.043)$ \\
C. varia & $0.026 \mathrm{a}$ & $147.8 \mathrm{a}$ & $0.117 \mathrm{a}$ \\
& $(0.013)$ & $(77.1)$ & $(0.049)$ \\
\hline
\end{tabular}

$29 \%$ reduction in total consumption, a $36 \%$ increase in instar duration, and a $9 \%$ increased in $\mathrm{AD}$ (it is likely that here, and throughout this study, increases in AD result directly from decreases in RCR and do not reflect increases in digestive efficiency per se). Larvae switched onto $M$. sativa from C. varia showed a $39 \%$ decrease in RGR, a $40 \%$ decrease in ECD, and a $41 \%$ decrease in pupal weight.

Similarly, larvae forced to switch onto $M$. alba during the ultimate instar, after feeding on either $M$. sativa or C. varia, performed significantly worse than larvae fed continuously on $M$. alba (Table $3 \mathrm{~b}$ ). Prior feeding on $M$. sativa resulted in a $73 \%$ decrease in $\mathrm{RGR}$, a $64 \%$ decrease in $\mathrm{ECD}$, a $31 \%$ decrease in RCR, a $50 \%$ decrease in total consumption, a $59 \%$ decrease in pupal weight, and a $17 \%$ increase in AD. Prior feeding on C. varia resulted in a $62 \%$ decrease in RGR, a $47 \%$ decrease in ECD, a $34 \%$ decrease in RCR, a $44 \%$ decrease in total consumption, a $53 \%$ decrease in pupal weight, and an $8 \%$ increase in AD.

In contrast to $M$. sativa and $M$. alba, C. varia supported adequate growth of larvae independent of their prior feeding experience (Table $3 \mathrm{c}$ ).

When the same data are arranged by common rearing diet instead of common test diet (Table $4 a-c$ ) it is evident that the induction of preference is advantageous, regardless of rearing diet. For larvae reared on $M$. sativa, RGR during the ultimate instar was $220 \%$ higher on $M$. sativa than on M. alba and $38 \%$ higher than on C. varia (Table $4 a$ ). For larvae reared on $M$. alba, RGR during the ultimate instar was $173 \%$ higher on $M$. alba than on M. sativa and $53 \%$ higher than on C. varia (Table 4b). For larvae reared on C. varia, RGR during the ultimate instar was $34 \%$ higher on C. varia than on $M$. sativa and $88 \%$ higher than on M. alba (Table 4c).

\section{Discussion}

Natural populations of Colias philodice commonly use several available host species (Watt et al. 1979; Stanton 1982, 
1983; Karowe 1988). This study provides evidence that such populations are likely to consist of several distinct types of phenotypically-specialized individuals, and suggests that, for $C$. philodice, polyphagy at the species or population level does not arise from polyphagy at the individual level.

As a consequence of prior feeding experience, fifth instar C. philodice larvae become physiologically and behaviorally restricted to a particular host species. Although all three legume species used in this study are equally acceptable to newly-emerged larvae, by the onset of the ultimate instar, C. philodice larvae show a strong induced preference for their particular rearing host. In general, larvae preferred their rearing host at least 2:1 over either alternative host. This behavioral specialization is highly advantageous. Regardless of rearing diet, switching to a novel host during the ultimate instar in all cases resulted in lower relative growth rate (RGR) and smaller pupal weight, both of which are significantly positively correlated with fecundity in C. philodice (Karowe 1988). If forced to switch to a novel host during the ultimate instar, larvae on all three plant species incurred a high physiological cost, as evidenced by reduced relative growth rate (RGR), relative consumption rate (RCR), conversion efficiency (ECD), and pupal weight, and increased instar duration. Thus, induction of feeding preference behaviorally predisposes C. philodice larvae to feed upon the host species they are most capable of utilizing for growth. Such within-generation specialization may explain in part why $C$. philodice females lay eggs singly rather than in clusters; laying eggs singly could decrease the likelihood that the original host will be defoliated and, therefore, that larvae will be forced to switch host species.

The overriding importance of ECD in determining RGR indicates a strong physiological component to within-generation dietary specialization and suggests two primary mechanisms of physiological specialization. First, larvae may become physiologically and biochemically acclimated to the nutrient content (e.g. the amino acid composition) of a particular host, such that maximal growth is no longer possible on a different host. Second, physiological specialization may result from costs, including the induction of new detoxification systems, associated with processing the secondary compounds of hosts not previously encountered. Interspecific diversity in secondary chemistry is particularly high within the Fabaceae (Harborne et al. 1971; Allen and Allen 1981).

Although M. sativa is a primary host of C.philodice and the most abundant host in southern Michigan, larvae fed only $M$. sativa did not grow significantly faster, attain higher pupal weight, or require less time to complete the fifth instar than larvae fed only $M$. alba or C. varia (compare Table $3 \mathrm{a}-\mathrm{c}$ ). In fact, both RGR and pupal weight tended to be higher on $M$. alba than on $M$. sativa. Why, then, is $M$. alba not the primary host for $C$. philodice? One possible answer was evident in this study. Despite higher RGR, larvae fed $M$. alba required nearly $20 \%$ longer to complete the ultimate instar than did larvae fed $M$. sativa. In nature, extension of the larval period may result in higher mortality due to increased parasitism and predation (Feeny et al. 1985). In addition, other studies indicate that larval mortality is significantly higher, and egg-to-pupa growth rate is significantly lower, on $M$. alba than on $M$. sativa (Karowe 1988).

Results of the present study agree with previous work on lepidopteran herbivores indicating that some, but not all, host-switches result in reduced growth. For instance, RGR of the Lauraceae specialist, Papilio troilus L., was reduced by forced switching between sassafras and spicebush (Scriber 1979), and ECD of Pieris rapae L. on cabbage was reduced by prior feeding on either nasturtium or a different variety of cabbage (Schoonhoven and Meerman 1978). However, RGR of Spodoptera eridania Cramer on black cherry was unaffected by prior feeding on either mountain ash or paper birch, and RGR on paper birch was actually increased by prior feeding on mountain ash (Scriber 1982). Scriber (1981) measured an increased metabolic cost associated with processing a mixed diet in S. eridania, but only if plant species were presented in a specific order. In the present study, the magnitude of adverse effects of host-switching depended on the host species. In particular, host-switching from $M$. sativa to $M$. alba or vice versa resulted in much stronger adverse effects than switching from C. varia to either of these hosts. Moreover, while prior feeding on C. varia resulted in decreased ability to use both $M$. sativa and $M$. alba, prior feeding on either $M$. sativa or $M$. alba did not result in decreased ability to use C. varia.

Finally, it is important to note that while this study demonstrates that behavioral specialization is advantageous once physiological specialization has occurred, it does not address whether physiological specialization itself is advantageous. The greater growth of unswitched than switched larvae could have arisen because (1) prior feeding on a given host species results in increased physiological ability to use that species, (2) prior feeding on a given host species results in reduced physiological ability to use alternative species, or both. Physiological specialization will be advantageous only if larvae reared on a single host use that host more efficientiy than do larvae reared on a mixed diet. This question will be addressed in future work.

Acknowledgements. I am grateful to J. Cresswell, M.M. Martin, B.J. Rathcke, H. Van't Hof, W.H. Wagner, and J. Witter for their helpful comments on an earlier version of this manuscript. This research was supported by NSF grant BSR-8421268 to M.M. Martin and by a Grant-In-Aid-of-Research from Sigma Xi, the Scientific Research Society.

\section{References}

Ahmad S (1983) Mixed-function oxidase activity in a generalist herbivore in relation to its biology, food plants, and feeding history. Ecology 64:235-243

Allen ON, Allen EK (1981) The Leguminoseae. University of Wisconsin Press, Madison

Barbosa P, Greenblatt, J. Withers W, Cranshaw W, Harrington E (1979) Host-plant preferences and their induction in larvae of the gypsy moth, Lymantria dispar. Entomol Exp Appl $25: 180-188$

Brattsten LB, Price SL, Gunderson CA (1980) Microsomal oxidases in the midgut and fatbody of a broadly herbivorous insect larvae, Spodoptera eridania Cramer (Noctuidae). Comp Biochem Physiol 66 C:231-237

Cassidy MD (1978) Development of an induced food plant preference in the Indian stick insect, Carausius morosus. Entomol Exp Appl 24:87-93

Dethier VG (1980) Food-aversion learning in two polyphagous caterpillars, Diacrisia virginica and Estigmene congrua. Physiol Entomol 5:321-325

Feeny P, Blau W, Kareiva P (1985) Larval growth and survivorship of the black swallowtail butterfly in central New York. Ecol Monogr 55:167-187 
Fox LR, Morrow PA (1981) Specialization: species property or local phenomenon? Science 211:887-893

Gould F (1979) Rapid host range evolution in a population of the phytophagous mite Tetranychus urticae Koch. Evolution $33: 791-802$

Gould F (1983) Genetics of plant-herbivore systems: interactions between applied and basic study. In: Denno RF, McClure MS (eds) Variable Plants and Herbivores in Managed and Natural Systems. Academic Press, New York, pp 599-653

Grabstein EM, Scriber JM (1982) Host-plant utilization by Hyalophora cecropia as affected by prior feeding experience. Entomol. Exp Appl 32:262-268

Hanson FE (1976) Comparative studies on the induction of food choice preferences in lepidopterous larvae. Symp Biol Hung $16: 71-77$

Harborne JB, Boulter D, Turner BL (1971) Chemotaxonomy of the Leguminosae. Academic Press, New York

Hare JD, Kennedy GC (1986) Genetic variation in plant-insect associations: survival of Leptinotarsa decemlineata populations on Solanum carolinense. Evolution 40:1031-1043

Ishaaya I, Swirski E (1976) Trehalase, invertase, and amylase activities in the black scale, Saissetia oleae, and their relation to host adaptability. J Insect Physiol 22:1025-1029

Jaenike J, Grimaldi D (1983) Genetic variation for host preference within and among populations of Drosophila tripunctata. Evolution 37:1023-1033

Jermy T, Hanson FE, Dethier VG (1968) Induction of specific food preference in lepidopterous larvae. Entomol Exp Appl $11: 211-230$

Karowe DN (1988) Ecological and evolutionary determinants of larval host plant range in the sulfur butterflies, Colias philodice and Colias interior. Ph.D. thesis, The University of Michigan

Klots AB (1951) A Field Guide to the Butterflies. Houghton Mifflin Company, Boston

Mitter C, Fuuyma DJ, Schneider JC, Hare JD (1979) Genetic variation and host plant relations in a parthenogenetic moth. Evolution: $33: 777-790$

Papaj DR (1986) Conditioning of leaf-shape discrimination by chemical cues in the butterfly, Battus philenor. Anim Behav 34:1281-1288

Schmidt DJ, Reese JC (1986) Sources of error in nutritional index studies of insects on artificial diet. J Insect Physiol 32:193-198

Schoonhoven LM, Meerman J (1978) Metabolic cost of changes in diet and neutralization of allelochemics. Entomol Exp Appl $24: 489-493$

Scott JA (1986) The Butterflies of North America. Stanford University Press, Stanford

Scriber JM (1979) The effects of sequentially switching foodplants upon biomass and nitrogen utilization by polyphagous and stenophagous Papilio larvae. Entomol Exp Appl 25:203-215

Scriber JM (1981) Sequential diets, metabolic costs, and growth of Spodoptera eridania (Lepidoptera: Noctuidae) feeding upon dill, lima bean, and cabbage. Oecologia 51:175-180

Scriber JM (1982) The behavior and nutritional physiology of southern armyworm larvae as a function of plant species consumed in earlier instars. Entomol Exp Appl 31:359-369

Stanton ML (1982) Searching in a patchy environment: foodplant selection by Colias p. eriphyle butterflies. Ecology $63: 839-853$

Stanton ML (1983) Short-term learning and the searching accuracy of egg-laying butterflies. Anim Behav 31:33-40

Via S (1986) Genetic covariance between oviposition preference and larval performance in an insect herbivore. Evolution 40:778-785

Waldbauer $\mathrm{G}$ (1968) The consumption and utilization of food by insects. Adv Insect Physiol 5:229-288

Watt WB, Han D, Tabashnik BE (1979) Population structure of picrid butterflies. II. A 'native' population of Colias philodice eriphyle in Colorado. Oecologia 44:44-52

Yamamoto RT (1974) Induction of hostplant specificity in the tobacco hornworm, Manduca sexta. J Insect Physiol 20:641-650

Yu SJ, Ing RT (1984) Microsomal biphenyl hydroxylase of fall armyworm larvae and its induction by allelochemicals and host plants. Comp Biochem Physiol 78 C:145-152

Received February 15, 1988 